\title{
EYE SYMPTOMS DUE TO OSTEOMYELITIS OF THE SUPERIOR MAXILLA IN INFANTS
}

\author{
BY
}

\author{
Dr. E. MARX \\ L.EYDEN (HOLI..IND)
}

THE disease that nowadays is generally called osteomyelitis of the superior maxilla, and was formerly known as acute sinusitis of the antrum Highmori in newly-born infants, sometimes presents a number of symptoms that lead to an oculist being consulted. It is true that this is not a frequent occurrence, for during the past nine years I have only seen three children who were suffering from the above affliction. It is important, however, that the diagnosis be determined in these few cases, and seeing that ophthalmic literature, at least as far as I am aware, ignores this complex of symptoms altogether, I take the liberty of mentioning the histories of these three cases, and adding a few remarks.

i few years ago a child but three weeks old was brought to me with a conjunctivitis, a fistula in the region of the right lacrymal sac and yellowish discharge from the left nostril and the hard palate; this latter, however, I only heard of later, for it was not mentioned to me by the person who came with the child. I misconstrued the symptoms, and imagined that I had to deal with a case of running discharge from the lacrymal sac, although on applying pressure to that sac nothing flowed from the canaliculi, which, as is well known, is not always necessary, provided the fistula discharges sufficiently. It very quickly became apparent that the canaliculi were quite normal, so that the only treatment needed consisted in keeping the eye clean with an eye lotion, which soon cured the inflammation of the membranes, and the fistula, after the osteomyelitis of the maxilla had been stopped by the good services of the surgeon (Dr. Boevé).

With the second child (also a patient of Dr. Boevé) there was a discharge from the mouth, from both maxillae, the upper and lower eyelids were swollen and red on both sides, and there was also some chemosis. In the right lower eyelid a fistula opened and gave off pus containing staphylococci. The left lower eyelid was swollen, as also the region of the lacrymal sac. The conjunctivae of both were red and there was also a slight discharge, whilst here also the canaliculi appeared unobstructed. After a few days a fistula also burst in the left lower eyelid, perfectly symmetrical with that on the other side. I did not see this patient again after the fourth day. All I can add is that the general 
condition of the patient was fairly good, so that a complete cure probably ensued.

The third patient I have recently observed in Professor Zaaijer's surgical clinic. The child was admitted owing to a swelling of the palate and the alveolar process, discharge in the mouth and a fistula in the upper lip. It showed a fairly pronounced protrusion of the right eye that could not be measured exactly. The movements of the eye were apparently not reduced, but the veins in the fundus were swollen. The pupils appeared to be equally large and to react well to light. The tension was normal. Two days later the protrusion was less, but now the region of the lacrymal sac and the right lower eyelid appeared to be swollen and fixed, whilst the conjunctiva of the eyelids was swollen also, and showed signs of a slimy discharge containing yellow staphylococci, similar to those that could be cultivated from the pus from the mouth. Here also the canaliculi worked normally. A few days later all eye symptoms had disappeared, though the discharge from the maxilla continued. The child was finally cured.

The three cases here mentioned are examples of an affliction $\underset{N}{\mathbb{N}}$ which, as stated, is nowadays mostly called osteomyelitis of the superior maxilla in infants, a disease seldom encountered, but of which about 35 cases are known to literature. In order to bring these under review I have arranged them in tabular form, in which the eye symptoms have been allotted a separate column, as in this case these naturally interest us the most. Going by former reports and from my own experience, I will now try to sketch briefly the aetiology, symptomatology, and the progress of this disease, for which I have consulted principally the excellent monographs of Brown Kelly ${ }^{(1)}$ and François. ${ }^{(2)}$

After this general sketch, I shall be better able to draw attention to those points which are of special importance to the oculist.

As regards the aetiology, practically all writers are agreed that this osteomyelitis of the superior maxilla, just as that of the long bones, must be attributed to bacterial infection. As to the nature of the bacilli which here show their influence, we know but little, even if it is known from the investigations of Allard and Sicard ${ }^{(3)}$ that in the osteomyelitis of infants pneumococci and streptococci o play a much greater rôle than staphylococci, which in patients of $\tilde{N}$ more mature age become so important for the osteomyelitis. I $\underset{\omega}{\sim}$ must, however, here state that in two of my cases I have found $₹$ staphylococci, but there the discharge had already been present $\varrho$ for some time, so that the original flora may have been dispersed by the staphylococci.

Careful bacteriological investigation in cases that are in their first stage will be very necessary in future, for the table shows that very little attention has been paid to this question. 
The point now to be considered is how these bacilli, whichever they may be, can cause the infection of the maxilla; thus from whence they come, and where they enter the maxilla. As to the latter question, one must bear in mind that the female genital passages are the breeding places of many sorts of microbes which may eventually prove injurious to the child. Nevertheless, I find only one clear example $\mathrm{e}^{(4)}$ of this in the medical literature, in which case, however, it is not stated that the bacteria of the mother (in this case staphylococci and streptococci) and the child were the same. Further, one may regard as of aetiological importance an infection issuing from the glands or nipples of the breasts, in cases of breast-fed infants, for which perhaps the cases of Douglas and Lacasse, as also my third one, plead, where lymphangitis mammae and cracks in the nipple were notified.

These cases, however, were also not sufficiently investigated from a bacteriological point of view. In the third place one must, as in every osteomyelitis, always bear in mind the possibility of blood transmigration. I can, however, find no real example of this in the literary records, and thus one will often be compelled to state that the origin of the bacilli, which causes the inflammation of the superior maxillae of infants, is unknown, at least provided no further importance is attached to the small skin wounds and ecchymoses, as in the cases of $\mathrm{W}^{\text {ood }}$ and H. Dujardin.

As regards the point at which the bacilli enter the maxilla, neither from the accounts collected in the literature, nor from $\mathrm{my}$ own experience (none of which is sufficiently precise) have I been able with any definite degree of certainty to determine this point.

Most of the writers who report on this are of the opinion, however, that the alveolar border is the spot where the bacilli enter, although often the swelling of the front side of the maxilla, with accompanying swelling and redness of the cheek, and various eye symptoms, are the first signs of the inflammation. This supposition that the place of access is in the alveolar process appears to me perfectly feasible, because the mouth offers the best opportunity for the invasion of bacilli. If one adheres to that supposition, then it is improbable that the bacilli enter through the perfectly sound mucous membrane, and one must thus assume small superficial abrasions : and then it is obvious that the alveolar process must be considered the most easily wounded spot in the maxilla.

The small wounds in the mucous membrane may originate in a birth trauma, i.e., owing to a narrow pelvis, or by pressure from the forcens, or by a face delivery where the fingers of the 
accoucheur must of ten go into the mouth. Landwehrmann ${ }^{(5)}$ has, moreover, drawn attention to the fact that in cleansing the mouth small wounds may be inflicted.

Having once settled in the maxilla the bacilli develop further in the spongiosa of the bone, without the antrum Highmori, which at birth is but very small, assisting in the inflammation. It was formerly supposed that this cavity was the point of exit of the inflammation, but Schmiegelow, ${ }^{(6)}$ Lichtwitz, ${ }^{(7)}$ and specially Brown Kelly, have shown that here one has to deal with a real osteomyelitis of the bone. In proof of this conception the writers just mentioned produce anatomical grounds (at birth the antrum is but a few square millimeters in size), as well as clinical ones, whereby, in my opinion, their contention is amply and finally proved. For the rest I will not venture any opinion on this question of surgical anatomy, which I myself have not been able to investigate.

Once the inflammation in the spongiosa has commenced, it goes further, just as in the osteomyelitis of the long bones. A heavy flow of blood to the inflamed parts sets in, followed by a discharge, and finally necrosis and sequestrum formation. If the matter has collected under the periosteum, then this is eaten into and pierced, and the inflammatory products can disperse in various directions.

The phenomena which the children hereby show are mostly fever, lack of appetite, and sometimes disturbances of the bowels. Usually swelling of the eyelids soon appears, often a fistula in the region of the lacrymal sac, inflammation of the conjunctiva and sometimes exophthalmus, in addition to a frequent discharge of matter from the nose, swelling and fistulas of the alveolar process and the palate, falling out of the teeth germs and sequestration. These symptoms appear in the most irregular sequence and combination, and some, as for instance, discharge from the nose and canine fossa, continue for a protracted period.

If the children are sturdy, and treatment is commenced at once, the prognosis is usually good. From the fact that death followed in more than 25 per cent. of the cases, one can deduce that these two conditions to a favourable result are not complied with by a long way.

The number of practically certain cases of osteomyelitis of the superior maxilla that I have been able to collect amounts to 35 , a very small number, though one must assume that many more occur than are published. In the column of the bones, compiled according to the frequency with which they were attacked by osteomyelitis, the superior maxilla, at least as far as adults are concerned, stands almost at the bottom. In infants, however, 
that bone occupies a much higher position. Why it happens that in very young children such inflammations are more frequent is perhaps apparent from the experiments of Leber, who showed that it is just in the full-blooded, rapidly growing bones that the bacilli attach themselves the most readily, and there develop. One must thus assume that the superior maxilla of infants grows very rapidly, which for the time being, so far as I am aware, is nothing more than a hypothesis.

Having thus sketched the general progress of this affliction, I can now devote myself more particularly to the eye symptoms, in the first place with regard to the number of times these appear.

Of the 35 cases here tabulated, 30 developed eye symptoms, and it is not certain that the remaining 5 did not have them, for, as I stated, the records of these cases are not complete, and one can be almost sure that when, for instance, a swelling of the cheek is recorded, the eyelids also will have been swollen.

As regards the time of the appearance of the eye symptoms, my information is probably not quite exact, because, as is very natural, former writers paid but little attention to this point. In 8 of the records, however, I have cause to think that it was the eye symptoms that attracted the attention of the family or the doctor. This is no small percentage, and therefore an important point for us, because it may happen that the oculist is the first to be consulted, and the further progress of events may therefore depend on his being able to arrive at a correct diagnosis. That this is not always the case is but too apparent in the last column of the table. Of the 35 cases, the results of 7 are not known: in 18 cases the patients were cured, whilst 10 died. This unfavourable record appears to a large extent to be attributable to surgical assistance being called in too late. Medical attention as early as possible improves the prognosis considerably, and therefore it is of the utmost importance that the oculist recognises the disease quickly. Examples where this has not been the case are to be found in one of my own records, and in that of Dujardin. ${ }^{(8)}$ The latter treated a child with red and swollen eyelids and pronounced exophthalmus on the left. As the condition got worse he made an incision in the eye socket, after which much discharge came and the condition quickly improved. The writer says : "A curious thing, matter came from the mouth and nose on the same side," but he.does not proceed any further with this phenomenon. In my opinion, this was an osteomyelitis of the superior maxilla with purulent discharge in the eve socket, indeed a most unusual process, and one which I have only seen recorded twice.

The eye symptoms which appear in this osteomyelitis of the superior maxilla can, for simplicity's sake. be divided into those 
Osteomyelitis of the Superior Maxilla in Infants 31

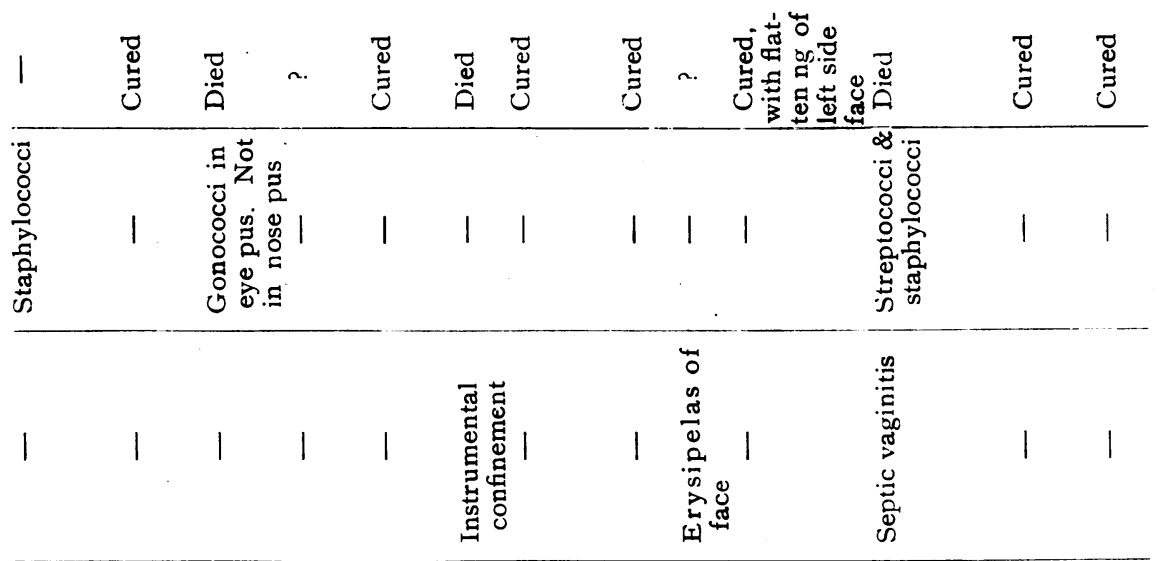

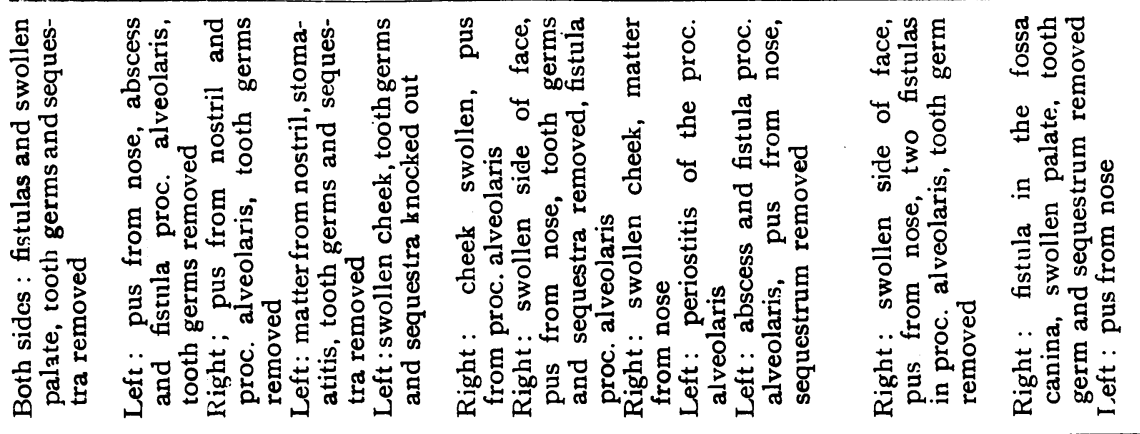

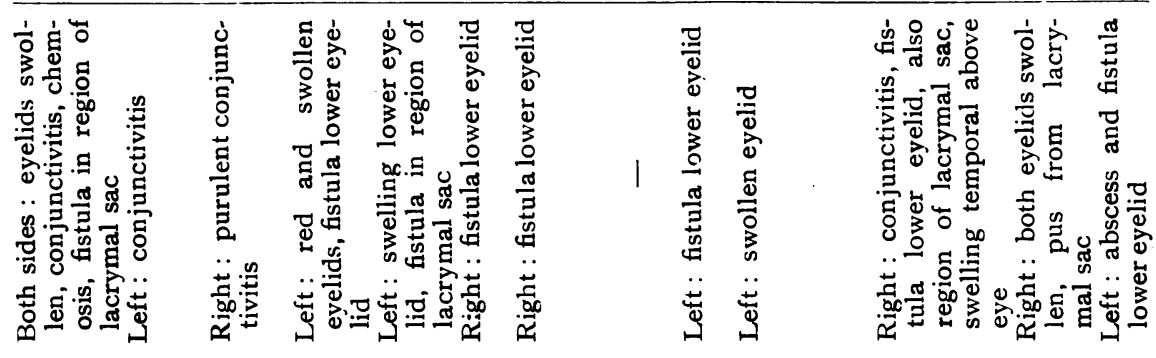

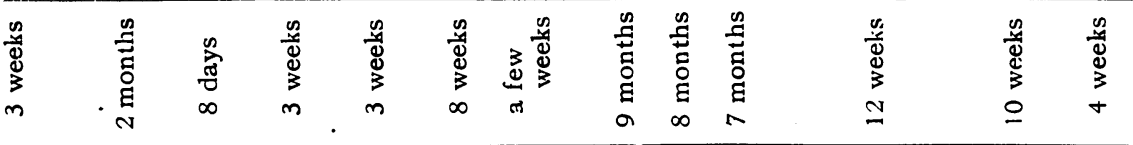

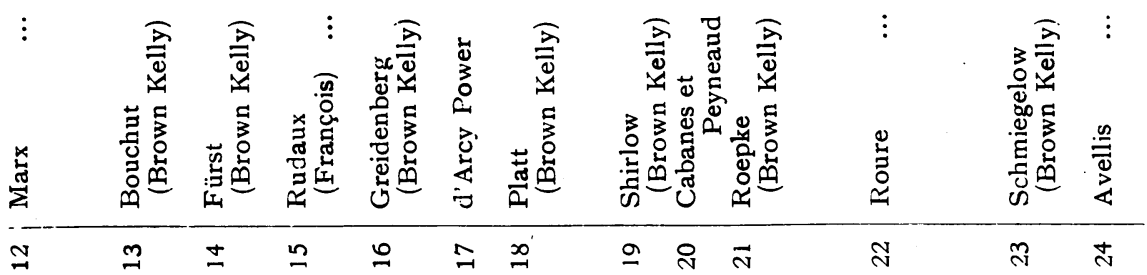


The British Journal of Ophthalmology

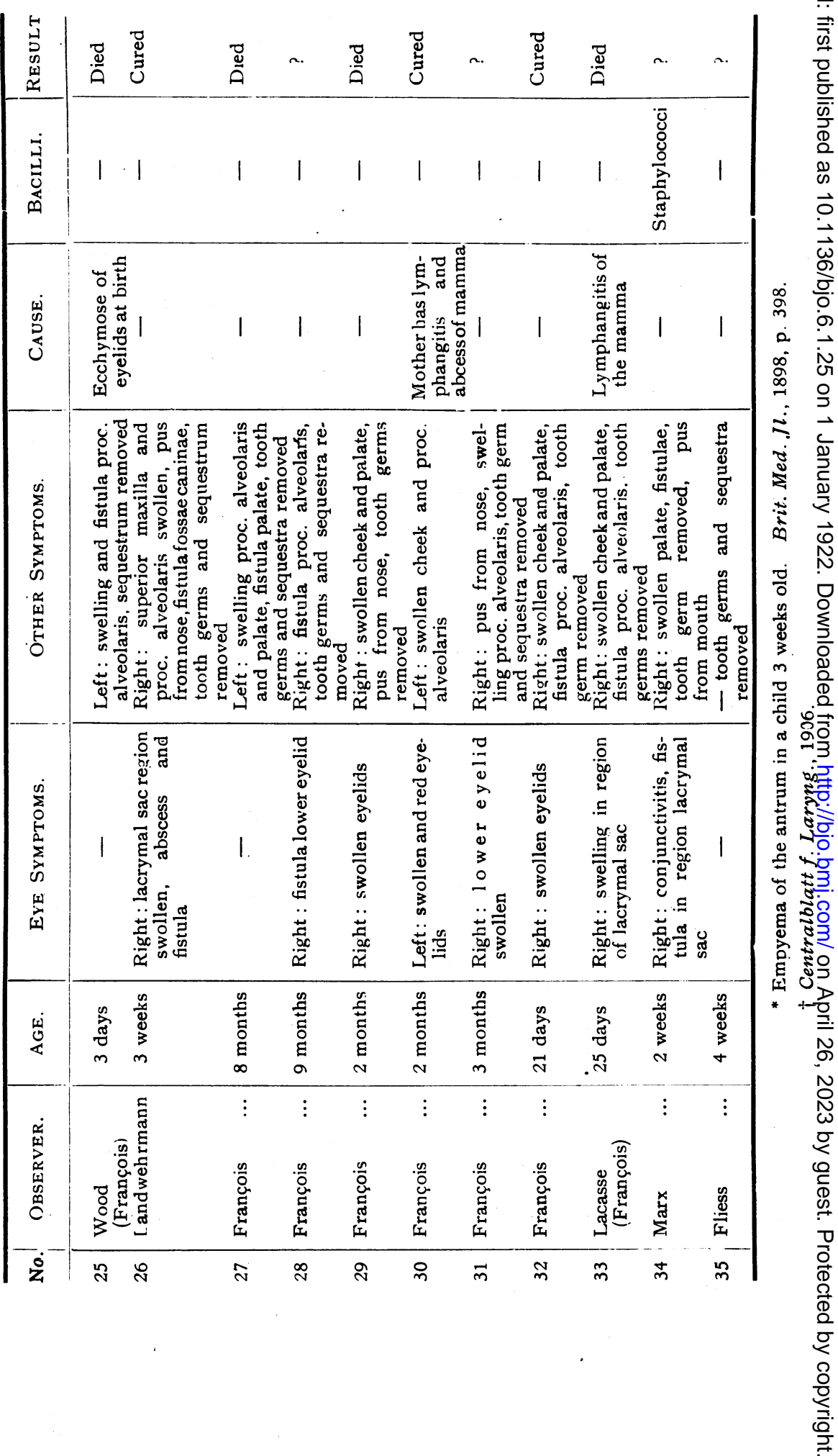


of the eyelids, of the conjunctiva, and, of the eye socket. The symptoms in the eyelids, according to Brown Kelly, are those which first attract the attention in the whole process. In the records at my disposal, I have, as already stated, only seen these symptoms mentioned as being the first to appear, in 8 cases, so that on this point no concurrence exists. The appearance of this swelling, owing to the severe inflammation of the maxilla is, however, as collateral oedema, quite easy to understand.

The swelling and redness in the region of the lacrymal sac and the frequent subsequent fistula are symptoms to which I wish to draw attention, because they may easily lead to an incorrect diagnosis. It is amazing, as Brown Kelly says, that an abscess often forms in the lower eyelid, and that pus can exude from the lacrymal sac. François expresses himself more cautiously when writing that the discharge from the fistula in the inner corner of the eye causes one to think of dacryocystitis, but that accompanying symptoms at once lead the diagnosis in another direction. The fistula just at this point is to be explained by the fact that the inflammation often prematurely develops in the ascending branch of the superior maxilla, and the pus collects there, eventually seeking the easiest method of exit, which, in this case leads to the inner corner of the eye. It is very remarkable that in not a single one of the 35 cases mentioned here is any remark made regarding treatment of the lacrymal sac, which, in all these cases, has apparently remained unaffected. In my three cases investigation shows that the canaliculi were really untouched, and it is well to remember this, because one might be too zealous, and, to the patient's detriment, begin an active treatment of the canaliculi if one did not pay proper attention to the other symptoms. I can only advise one to be as conservative as possible, and even in the commencement of abscess formation in the lower eyelid to make no incision in the abscess, as frequently treatment viâ the palate and processus alveolaris will (as in my case) effect a complete cure, whilst an ugly drawn scar with ectropion may result from incision, as appears from the report of Avellis. ${ }^{(9)}$ It is otherwise not surprising that the discharge from the maxilla in infants does not go over to the lacrymal sac, for it is well known that diseases of the lacrymal ducts in adults also depend far more on inflammation of the ethmoid labyrinth than the antrum Highmori. ${ }^{(10)}$ I do not doubt that in an isolated case inflammation of the lacrymal ducts in the disease in question will be present, but going by experience so far obtained that will not be frequent.

The conjunctivitis, like the swelling of the eyelids, must be taken as a sign of collateral oedema. The degree of severity of the 
original affection can thereby appear to a certain extent from the $\stackrel{\overrightarrow{\vec{s}}}{\stackrel{\vec{\rho}}{\vec{\theta}}}$ chemosis, which I only observed in the first acute period.

Finally, there remains the discussion of the exophthalmus, which was observed in 10 of the 35 cases. In comparison with the former eye symptoms, which were practically regular symptoms of the disease, this appeared too seldom to be regarded as a sure part of the disease. Exophthalmus, however, is a certain accompaniment of inflammation of the ethmoid. Stephenson ${ }^{(1)}$ has reported 10 cases of inflammation of the ethmoid, which all showed exophthalmus to a greater or lesser degree. Among these 10 there was a 14 days' old child who showed this prominence of the eye in a marked degree, whose eyelids were oedematous, and who suffered from a discharge from its nose. After an incision in the o eye socket pus appeared and the child was soon cured. Another similar case is mentioned by Harry, ${ }^{(12)}$ which refers to a child of 3 weeks that also came under treatment with severe exophthalmus. Curettage of the anterior ethmoid sinuses caused pus to $\underset{\gtrless}{2}$ appear, after which the child soon got better. Thus it appears $\overrightarrow{0}$ that the protrusion of the eye is attendant on the inflammation of $\underset{N}{\mathbb{N}}$ the ethmoid cells, which apparently is always followed by purulent exudation in the eye socket. This, again, is further explained by the case of van der Wildenberg in my table, in which in a suppurative jaw affection in which exophthalmus was also present, and in which during the treatment ethmoid cells filled with pus, were opened. This report appears to point out that if during an inflammation of the jaw the eye is pressed forward the ethmoid is also affected. In my opinion, children may suffer from genuine jaw inflammations, genuine ethmoid inflammations, and forms comprising both, each of which must be considered if a protrusion of the eye accompanies a maxilla osteomyelitis.

From this short explanation I think it seems quite clear that the jaw discharges in infants have a certain importance for the oculist. It is, therefore, very surprising that in the ophthalmic literature practically nothing is to be found about these affections. I have only come across one reference by Eversbusch (Die Augenerkrankungen im Kindesalter). The writer points out the possibility of mistaking the discharge from the lacrymal ducts for outbreaking osteomyelitis of the superior maxilla. Further, $\tilde{N}$ I have not been successful in finding other references, which are $\omega$ nevertheless necessary, as our knowledge regarding these inflammatory conditions is in many places insufficient, especially as regards the aetiology, the progress of the disease, and the exophthalmus.

Here also is again a ground for fruitful combination between various specialities. 


\section{REFERENCES}

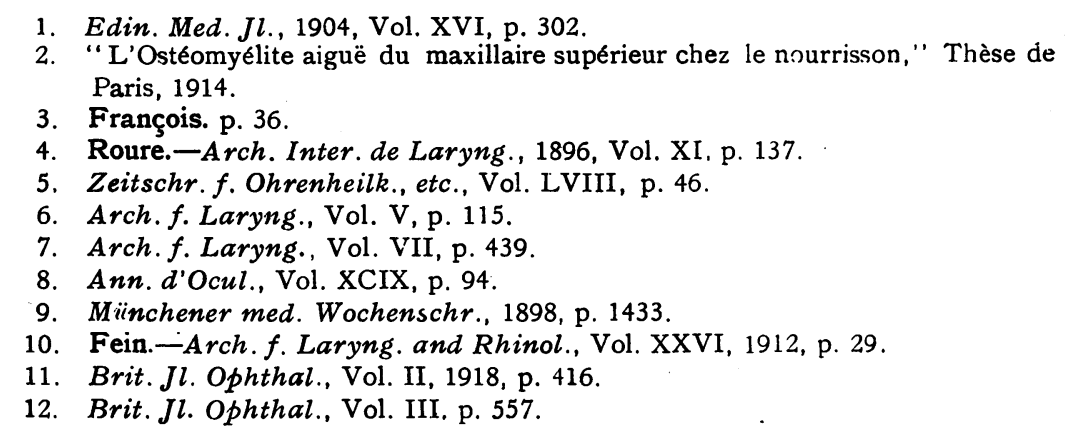

\section{MEGALOPHTHALMUS AND MICROPHTHALMUS}

BY

R. E. Wright, M.D., Major, I.M.S.

MADRAS

IN the Transactions of the Ophthalmological Society of the United Kingdom, 1920, Vol. XL, p. 132 et seq., will be found an interesting communication by $\mathrm{Mr}$. Treacher Collins on megalocornea and microcornea, in which the conditions known as megalocornea, megalophthalmus, hydrophthalmus congenitus, microcornea, nanophthalmia, and microphthalmus are referred to.

As some of these conditions are of great interest and rarity, and as I am not certain as to what class of case Seefelder (quoted by Mr. Collins), includes under the term megalophthalmus, I feel that it is not out of place to publish the following notes.

Buphthalmus (infantile glaucoma, hydrophthalmus congenitus) is comparatively frequent in South India, and although I have come across cases with comparatively good vision and normal tension, with practically symmetrical eyes, I have never seen a case in which there was not considerable deep corneal opacity, very obvious on examination with loupe or microscope. In the beginning of this year a case was met with which did not give the impression of hydrophthalmus congenitus, nor yet did it comply with the conditions which, as stated by Seefelder, distinguish Megalocornea. The case may perhaps be referred to as one of megalophthalmus.

Brief notes are as follows:-

D. S., Mahomedan, aged 45, admitted 22/3/21 for cataract B.E. 